\section{Pase Oreparts}

\section{Endovascular management of RFA- induced hepatic arterial injury}

\section{Introduction}

Radiofrequency ablation (RFA) is a widely accepted alternative treatment for unresectable primary and metastatic hepatic tumours with favourable survival and excellent local control rates. ${ }^{1}$ We describe the case of a patient with HCC who underwent ultrasound-guided RFA of a subcapsular lesion followed by vascular injury leading to haemoperitoneum which was managed by successful intra-arterial embolization of the bleeder.

\section{Case report}

A 65-year-old woman was referred for cytoreductive therapy for HCC. A biphasic, contrast-enhanced computed tomography (CECT) abdomen showed cirrhotic changes in the liver with presence of two hypervascular lesions in segments V and VIII measuring $1.4 \times 1.2 \times 1.2 \mathrm{~cm}$ and $2.8 \times 2.5 \times 2.2 \mathrm{~cm}$, respectively (Figure 1). There was portal hypertension with presence of ascites. Both the lesions were subcapsular in location. No distant metastases were present. A decision to first ablate the larger segment VIII lesion was taken despite the presence of ascites. RFA was carried out using a bipolar "cool tip" electrode (Valleylab, US) and three cycles of therapy of 12 minutes' duration each were given under ultrasound guidance (Figure 2A). The patient complained of chest discomfort towards the end of the procedure. As she was being monitored under conscious sedation, cardiac arrhythmia was noticed which was corrected by the anaesthetist. The smaller lesion was thus left to be tackled at a later session. After 6-8 hours, the patient began complaining of abdominal pain associated with progressive abdominal distension. Haemogram showed a fall in the haematocrit level and bedside sonography confirmed the presence of haemoperitoneum. CT angiography was immediately done which showed few tortuous vessels in relation to the ablated lesion with perihepatic haematoma and haemoperitoneum (Figures 2B, 2C). Digital subtraction angiography (DSA) revealed a small lobulated pseudoaneurysm arising from one of the branches of the anterior division of the right hepatic artery (Figures 3A, 3B). Selective embolization
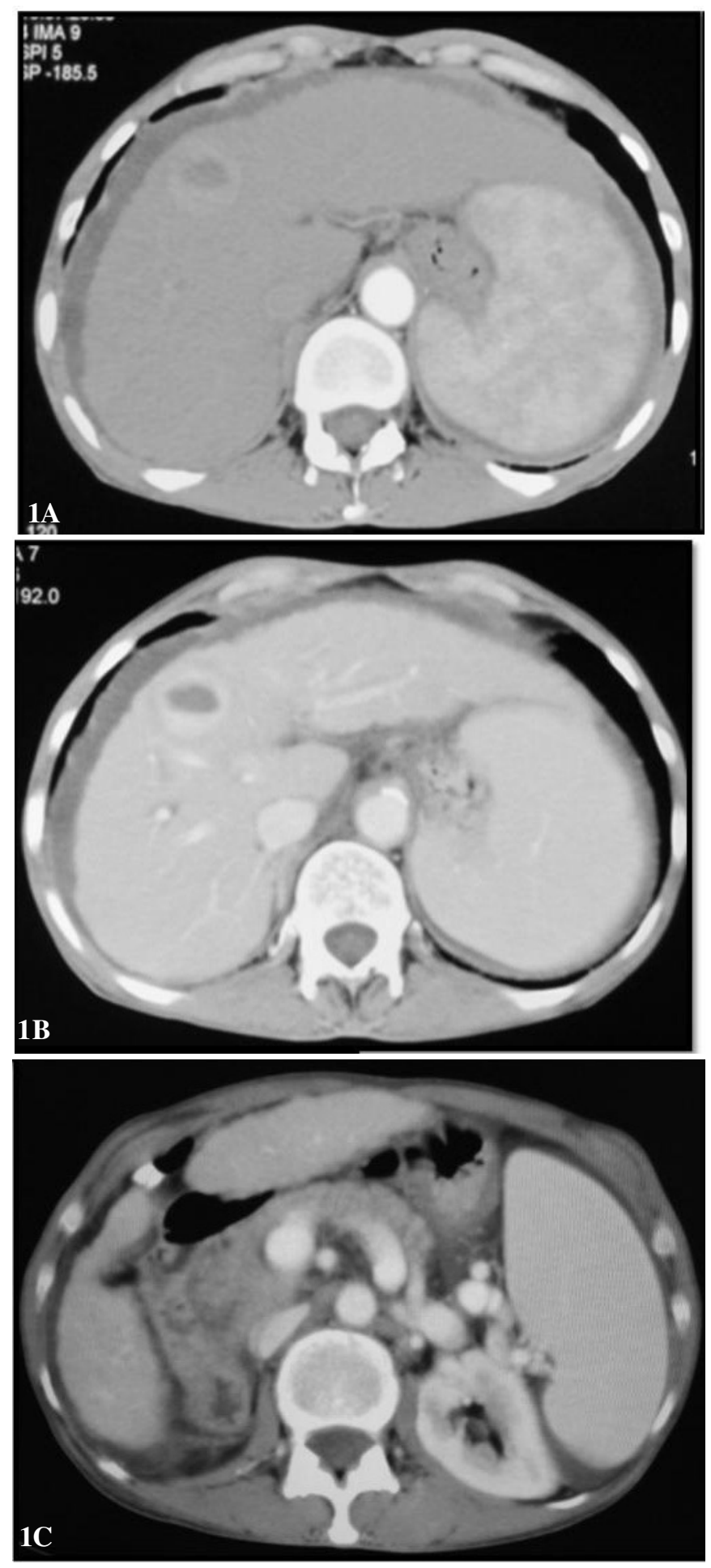

Figure 1: Biphasic, contrast-enhanced computed tomography (CECT) abdomen showing hypervascular lesions in segments VIII (A,B) and V (C) (arrow). Note changes of chronic liver disease and portal hypertension.

of the implicated artery was performed using a mixture of gelfoam and polyvinyl alcohol (PVA) particles, the latter ranging in size 300-500 micron. Microcoils were not available during that emergency. Complete occlusion of the artery was achieved. During the procedure, it was noticed that the smaller segment $\mathrm{V}$ lesion was also receiving supply from one of the adjacent 

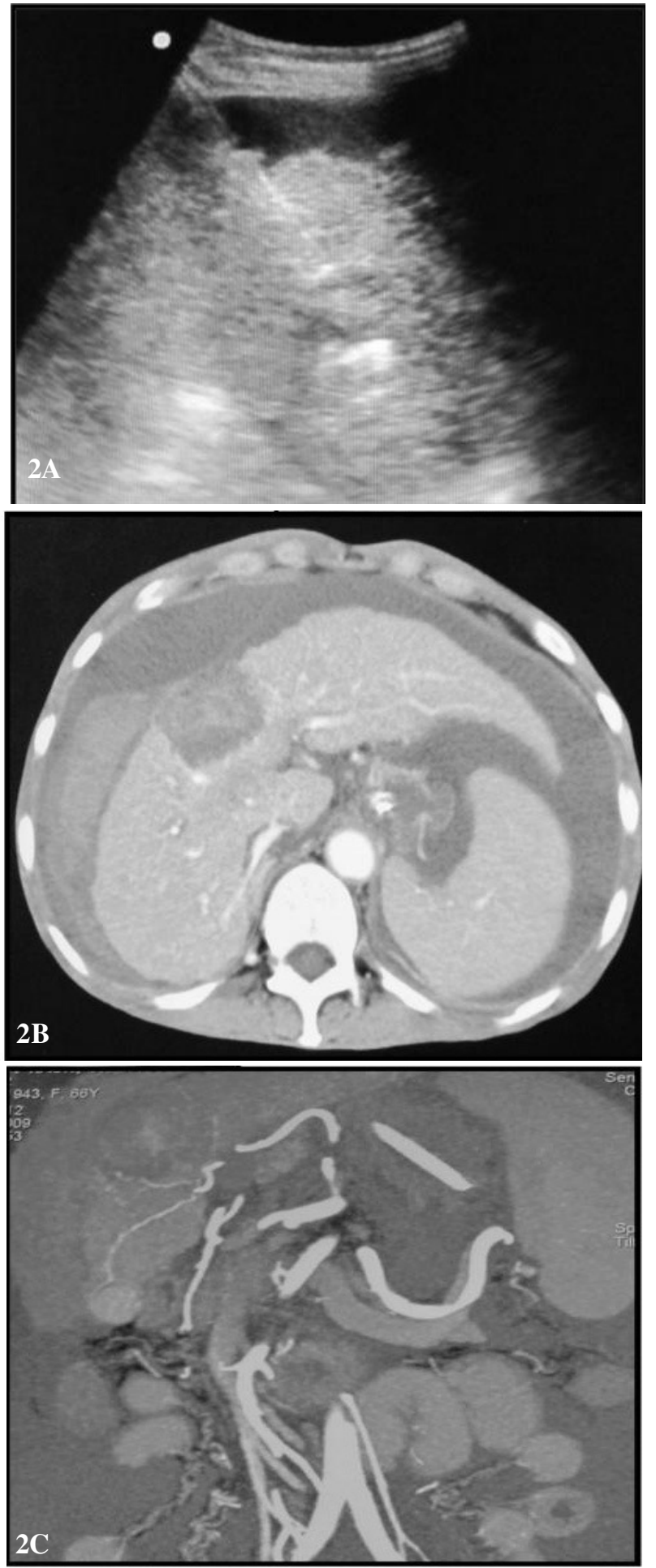

Figure 2: (A) Radiofrequency ablation (RFA) electrode in situ (arrow) within the segment VIII HCC. Note the perihepatic fluid. (B) Axial section of arterial phase contrast-enhanced computed tomography (CECT) abdomen showing ablated HCC with presence of subcapsular haematoma and haemoperitoneum. (C) Coronal maximum intensity projection (MIP) image of the arterial phase depicting branches leading into the ablated lesion (arrowhead) and smaller HCC.

branch of the right hepatic artery (Figures 3A, 3B). After consultation with the treating hepatologist, a decision was

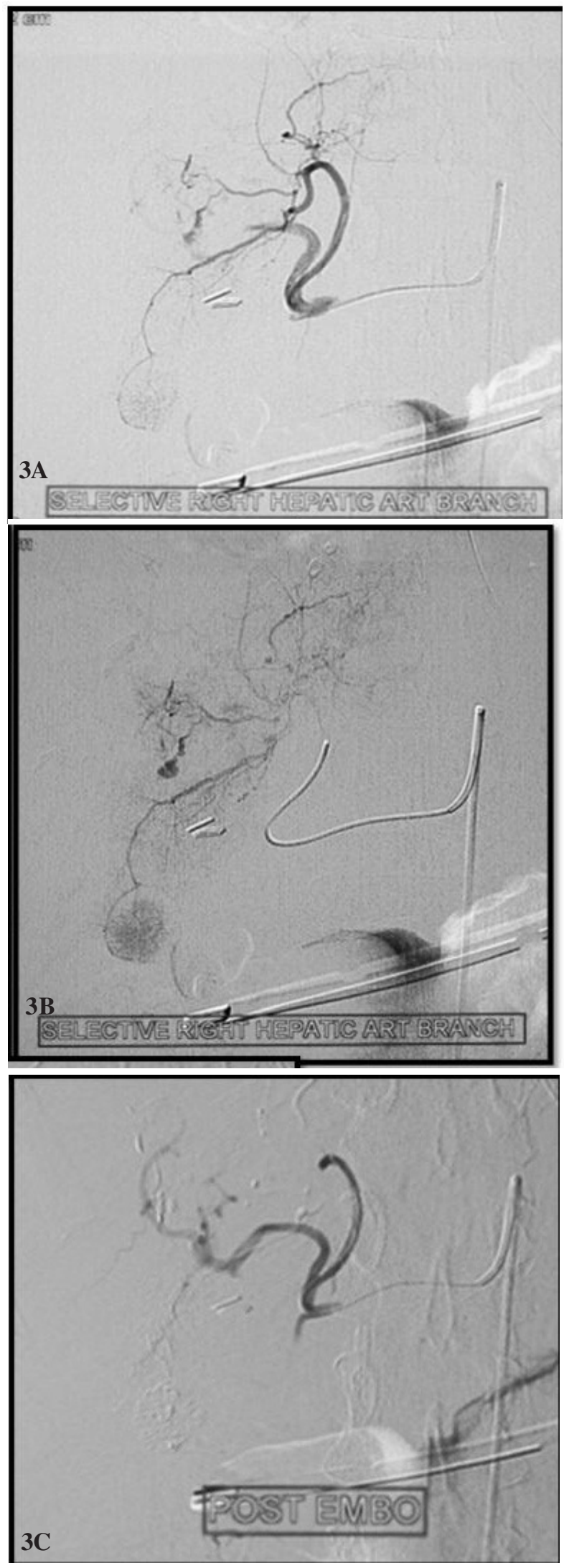

Figure 3: Selective right hepatic arteriogram depicting the pseudoaneurysm (arrowhead) and segment V HCC (arrow) (A,B). Check angiogram post-procedure (C) showing embolization of the pseudoaneurysm as well as segment V HCC. 
taken to embolize it with doxorubicin. The feeding artery was completely embolized using doxorubicin with lipiodol followed by gelfoam slurry (Figure 3C). The patient was under constant monitoring in the ICU for 2 days when she left against medical advice. However, she was haemodynamically stable at this time.

\section{Discussion}

Complications that may be encountered during or after an RFA procedure include bleeding, peritonitis and injury to the adjacent vessel or the large bowel; these demand immediate attention. ${ }^{2}$ Mechanical damage caused by the placement of electrodes appears to be the likely reason for bleeding, ${ }^{3}$ especially if the needle is placed directly in a superficially located lesion without traversing significant normal hepatic parenchyma. Coagulopathy is a major risk factor for haemorrhage after RFA. Thus, patients with cirrhosis having a deranged coagulogram are at a higher risk than those without cirrhosis. ${ }^{2}$ Other risk factors include attempt at multiple punctures or the use of multiple electrodes and the location of the targeted lesion behind a major blood vessel. Cauterization of the electrode tract during withdrawal may be an effective method for avoiding or reducing haemorrhage. ${ }^{4}$

Traditionally, the risk of bleeding in subcapsular lesions is considered to be greater in comparison to that due to deep seated lesions. Some authors ${ }^{5}$ have considered subcapsular location as a contraindication for percutaneous therapy of such lesions due to increased haemorrhage and recurrence rates. However, recent results ${ }^{6,7}$ have shown otherwise. Logic tells us that the presence of concomitant ascites would increase the risk of haemoperitoneum after RFA. The hypothesis put forward is that the ascitic fluid would wash away the thrombogenic material at the puncture site and reduce the "tamponade effect" from the opposing parietal peritoneum against the liver. ${ }^{8}$ This would thus increase the risk of sustained intraperitoneal haemorrhage from the hepatic surface in patients who have massive ascites as the tamponade effect would not occur. Fortunately, this potential complication related to free fluid in the peritoneal cavity is quite rare. For deep-seated or "invisible" hepatic tumours that require ablation creating artificial ascites is an established modality for easy visualization of these sonographic "occult" lesions before inserting the needle electrode. ${ }^{8,9}$ Also, artificial ascites helps in insulation and thus prevents the abdominal organs such as diaphragm and bowel loops from the heating effects of RF.

NAVEEN KALRA,

\section{CHIRAG KAMAL AHUJA, AJAY DUSEJA*, YOGESH CHAWLA*, NIRANJAN KHANDELWAL}

Correspondence: Chirag Kamal Ahuja Departments of Radiodiagnosis and Hepatology*, Post Graduate Institute of Medical Education and Research

Sector 12, Chandigarh.

Email:chiragkahuja@rediffmail.com

\section{References}

1. Tateishi R, Shiina S, Teratani T, Obi S, Sato S, Koike Y, et al. Percutaneous radiofrequency ablation for hepatocellular carcinoma. An analysis of 1000 cases. Cancer. 2005;103:1201-9.

2. Rhim H. Complications of radiofrequency ablation in hepatocellular carcinoma. Abdom Imaging. 2005;30:409-18.

3. Murphy FB, Barefield KP, Steinberg HV, Bernardino ME. CT or sonography-guided biopsy of the liver in the presence of ascites: frequency of complications. AJR Am J Roentgenol. 1988;151:485-6.

4. Pritchard WF, Wray-Cahen D, Karanian JW, Hilbert S, Wood BJ. Radiofrequency cauterization with biopsy introducer needle. $J$ Vasc Interv Radiol. 2004;15:183-7.

5. Komorizono Y, Oketani M, Sako K, Yamasaki N, Shibatou T, Maeda M, et al. Risk factors for local recurrence of small hepatocellular carcinoma tumors after a single session, single application of percutaneous radiofrequency ablation. Cancer. 2003;97:1253-62.

6. Filippousis P, Sotiropoulou E, Manataki A, Konstantinopoulos O, Thanos L. Radiofrequency ablation of subcapsular hepatocellular carcinoma: single center experience. Eur J Radiol. 2011;77:299-304.

7. Poon RT, Ng KK, Lam CM, Ai V, Yuen J, Fan ST. Radiofrequency ablation for subcapsular hepatocellular carcinoma. Ann Surg Oncol. 2004;11:281-9.

8. Rhim H, Lim HK, Kim YS, Choi D. Percutaneous radiofrequency ablation with artificial ascites for hepatocellular carcinoma in the hepatic dome: initial experience. Am J Roentgenol. 2008;190:91-8.

9. Song I, Rhim H, Lim HK, Kim YS, Choi D. Percutaneous radiofrequency ablation of hepatocellular carcinoma abutting the diaphragm and gastrointestinal tracts with the use of artificial ascites: safety and technical efficacy in 143 patients. Eur Radiol. 2009;19:2630-40.

\section{Calcified pancreatic pseudocyst}

\section{Introduction}

Pancreatic calcification commonly involves the pancreatic duct. Parenchymal calcification and calcification of pseudocysts are uncommon. 